\title{
Dismantling airway disease with the use of new pulmonary function indices
}

\author{
Sabine C. Zimmermann (1) 1 ,2,3,4, Katrina 0. Tonga (10) $1,2,3,4,5,6$ and \\ Cindy Thamrin (1) ${ }^{1,4}$ \\ Number 1 in the Series "Dismantling Airway Disease" \\ Edited by Sinthia Bosnic-Anticevich and Marc Humbert
}

Affiliations: ${ }^{1}$ Airway Physiology and Imaging Group, Woolcock Institute of Medical Research, The University of Sydney, Sydney, Australia. ${ }^{2}$ Dept of Respiratory Medicine, Royal North Shore Hospital, Sydney, Australia. ${ }^{3}$ Sydney Medical School Northern, The University of Sydney, Sydney, Australia. ${ }^{4}$ Woolcock Emphysema Centre, Woolcock Institute of Medical Research, The University of Sydney, Sydney, Australia. ${ }^{5}$ Dept of Thoracic and Transplant Medicine, St Vincent's Hospital, Sydney, Australia. ${ }^{6}$ Faculty of Medicine, The University of New South Wales, Sydney, Australia.

Correspondence: Cindy Thamrin, Woolcock Institute of Medical Research, 431 Glebe Point Road, Glebe NSW 2037, Australia. E-mail: cindy.thamrindasydney.edu.au

@ERSpublications

Spirometry is used to diagnose and manage airway disease such as asthma and COPD, but relates poorly to symptoms, lacks sensitivity and is effort dependent. FOT and MBNW are emerging clinical lung function tests that help us dismantle disease mechanisms. http://ow.ly/nM0G30nS6Ct

Cite this article as: Zimmermann SC, Tonga KO, Thamrin C. Dismantling airway disease with the use of new pulmonary function indices. Eur Respir Rev 2019; 28: 180122 [https://doi.org/10.1183/16000617.01222018].

ABSTRACT We are currently limited in our abilities to diagnose, monitor disease status and manage chronic airway disease like asthma and chronic obstructive pulmonary disease (COPD). Conventional lung function measures often poorly reflect patient symptoms or are insensitive to changes, particularly in the small airways where disease may originate or manifest. Novel pulmonary function tests are becoming available which help us better characterise and understand chronic airway disease, and their translation and adoption from the research arena would potentially enable individualised patient care. In this article, we aim to describe two emerging lung function tests yielding novel pulmonary function indices, the forced oscillation technique (FOT) and multiple breath nitrogen washout (MBNW). With a particular focus on asthma and COPD, this article demonstrates how chronic airway disease mechanisms have been dismantled with the use of the FOT and MBNW. We describe their ability to assess detailed pulmonary mechanics for diagnostic and management purposes including response to bronchodilation and other treatments, relationship with symptoms, evaluation of acute exacerbations and recovery, and telemonitoring. The current limitations of both tests, as well as open questions/directions for further research, are also discussed.

\section{Introduction}

Pulmonary function testing plays an important role in the clinical assessment of chronic airway disease, ranging from diagnosis to monitoring and management. To date, the gold standard test of lung function remains spirometry. In asthma, spirometry and peak flow are part of the formal diagnosis and assessment of disease and are used to demonstrate variable airflow limitation over time, combined with clinical symptoms such as wheeze, dyspnoea, chest tightness or cough [1]. In chronic obstructive pulmonary

Provenance: Submitted article, peer reviewed.

Received: Dec 222018 | Accepted: Feb 152019

Copyright $\odot$ ERS 2019. This article is open access and distributed under the terms of the Creative Commons Attribution Non-Commercial Licence 4.0. 
disease (COPD), spirometry is used to demonstrate persistent airflow limitation and define disease severity, in conjunction with respiratory symptoms like dyspnoea, cough or sputum production [2].

Despite this, spirometry has several shortcomings. For one, it often only has a weak relationship with patient-centred outcomes such as symptoms. It is also relatively insensitive in detecting early disease or people at risk of developing chronic airway disease. This is probably because spirometry primarily reflects bulk flow in the large airways. Although asthma and COPD are both highly heterogenous diseases, the small airways have been implicated in both conditions $[3,4]$. In asthma, small airways dysfunction can be demonstrated in more than half of patients, including those with mild disease [5]. While established COPD is known to affect both large and small airways [6], COPD is thought to arise from the small airways [7]. There is limited functional assessment of the small airways in clinical practice to date.

In addition, spirometry is effort-dependent, requiring forced expiratory manoeuvres that can be difficult or sometimes even impossible to perform for the very young, elderly or highly obstructed patients. Effort-independent and objective markers which are sensitive to both large and small airway mechanics would be a highly desirable addition to the currently available set of lung function tools for evaluating airway disease.

This article describes new pulmonary function indices available from two lung function tests, forced oscillation technique (FOT) and multiple breath nitrogen washout (MBNW), which provide us with an opportunity to examine the small airways and dismantle disease mechanisms. These tests have contributed much to our current understanding of airway disease, in particular asthma and COPD, which will be the focus of this article. They are also emerging as clinical tests, with increasing studies examining their clinical utility as measures of disease status, targets for treatment and predictors of future risk.

\section{Forced oscillation technique}

FOT is a noninvasive, objective and effort-independent method to measure airway mechanics. The key physical quantity measured by FOT is respiratory system impedance, which describes the relationship between pressure applied at the airway opening and the resultant flow, or vice versa. In FOT, pressure oscillations of low amplitude, commonly generated by a loudspeaker or a piston, are superimposed at the mouth, and can be measured during resting tidal breathing. Different oscillation signals are used in practice, with the most common ones being single or combinations of non-multiple and/or pseudorandom frequencies (conventional FOT), or harmonic multiples (impulse oscillometry). This article covers work involving both types of signals. The two main components of impedance measured by FOT are respiratory system resistance (Rrs) and reactance (Xrs), respectively. Rrs represents impedance to airflow changes, primarily reflecting a measure of overall airway calibre, whereas Xrs represents impedance to volume changes, and primarily encompasses the inertial and elastic properties of the respiratory system.

These measures are dependent on the frequency of the oscillations, revealing further insight into airway and lung mechanics. In most clinical studies, Rrs and Xrs are measured at (or close to) $5 \mathrm{~Hz}$, i.e. R5 and $\mathrm{X} 5$, where resistive and elastic properties dominate respectively. This is close to the resonant frequency of the respiratory system, at which point $\mathrm{X}$ becomes more dominated by inertial rather than elastic forces. Another frequently encountered measure in the literature is $\mathrm{AX}$, which represents the sum of Xrs components at all frequencies prior to resonant frequency. Furthermore, the difference in Rrs between 5 and $20 \mathrm{~Hz}$ (R5-R20) is used as an attempt to quantify frequency dependence in Rrs, although the interpretation of this index is often misunderstood. While often thought of as simply peripheral airway resistance, in reality it is a complex measure which also takes into account heterogeneity in ventilation/ mechanics across the airway tree and the upper airway shunt [8-10].

In addition to these two measures of "total" resistance and reactance, it is also possible to track changes in $R$ rs and Xrs within a breathing cycle. In doing so, FOT can be partitioned further into its inspiratory only (inspiratory resistance and reactance (Rinsp and Xinsp), respectively) and expiratory only (expiratory resistance and reactance (Rexp and Xexp), respectively) components, as well as derive a measure of expiratory flow limitation.

\section{Detection of expiratory flow limitation}

FOT has been shown to detect expiratory flow limitation (EFL), a phenomenon which occurs when increased expiratory effort and driving pressure do not result in increased corresponding flow, due to regional "choke points" from airway closure or narrowing in the distal airways. EFL can occur in asthma [11] and is a pathological hallmark in COPD, often manifesting as dynamic hyperinflation and increased exertional dyspnoea resulting in exercise limitation [6]. The presence of EFL has been found to predict the severity of breathlessness in COPD better than forced expiratory volume in $1 \mathrm{~s}$ (FEV1) [12, 13]. EFL may also represent a marker of disease severity and associated morbidity in terms of symptoms, functional 
impairment, degree of airflow obstruction and extent of gas trapping [14]. However, conventional methods of detecting EFL have either been invasive in nature (e.g. oesophageal balloon technique) or involve highly specialised operator and/or large inter-operator variability (e.g. the negative expiratory pressure technique). DeLLACÀ et al. [15] established a noninvasive method to detect EFL with high sensitivity and specificity, by applying within-breath FOT analyses and calculating the difference between the inspiratory and expiratory $X \mathrm{rs}$ at a frequency of $5 \mathrm{~Hz}$ (i.e. $\Delta X \mathrm{rs}=X i n s p-X \exp )$. When EFL develops, there is a sudden increase in the magnitude of Xexp, which possibly reflects expiratory small airway closure and which does not occur during inspiration. The method has shown potential clinical utility in titrating nasal continuous positive airway pressure (CPAP) treatment in COPD patients [16]. In further work, LorX et al. [17] used plots of the within-breath changes of FOT measures as functions of tidal flow and volume to visualise and differentiate between partial versus complete EFL.

\section{Relationship with symptoms, disease status and severity}

Spirometry consistently correlates poorly with symptoms like breathlessness [18-20]. In contrast, YounG et al. [10] demonstrated that Rrs and Xrs correlated with asthma control, and R5-20 with quality of life in COPD. R5-20 was additionally related to ventilation defects measured by magnetic resonance imaging in both asthma and COPD [10]. In addition, FranTz et al. [21] showed larger Rrs and more negative Xrs values in 450 subjects with self-reported symptoms suggestive of COPD, irrespective of whether spirometry was abnormal. Thus, in addition to demonstrating relationship with symptoms, FOT may also be better than conventional measures in the early detection of disease. FOT measures are also proportional to the degree of underlying airflow obstruction in COPD [22, 23]. In early or mild disease compared to never-smoking normal subjects, these changes are predominantly characterised by an increase in Rrs parameters, and in moderate to severe airflow obstruction by increasingly negative Xrs measures.

\section{Evaluation of bronchodilator/treatment response}

FOT may also be useful in measuring response to bronchodilation, and as outcome measures in the assessment of response to pharmacological treatment in clinical trials. Studies have shown R5 and X5 to be more sensitive than spirometric measures like $\mathrm{FEV}_{1}$ in detecting a bronchodilator response to short-acting $\beta$-agonist (SABA) administration in asthma [24], and to SABA [25-27] and long-acting $\beta$-agonist (LABA) [28] in COPD. The latter is notable since COPD is conventionally thought of as a disease of irreversible airflow limitation as measured via spirometry. DiBA et al. [29] found in moderate-to-severe COPD subjects that an improvement in respiratory system conductance (the inverse of Rrs) predicted the reduction in exertional dyspnoea experienced following bronchodilator (tiotropium bromide) administration, independent of improvements in spirometry or plethysmographic measures of hyperinflation. MiLne et al. [30] further demonstrated that in response to the LABA indacaterol, FOT indices correlated with hyperinflation and gas trapping. Importantly, FOT measures predicted the volume responses to indacaterol, suggesting FOT may be clinically useful to determine which COPD patients might benefit from bronchodilator treatment.

Other work suggests that FOT indices are responsive to combination treatment of inhaled corticosteroids (ICS) with LABA. For example, AKAmatsu et al. [31] showed that FOT predicted improvement in FEV1 following 2 months of ICS/LABA therapy in untreated asthma. In patients with moderate COPD, TIMmINS et al. [32] demonstrated significant improvements in FOT measurements coupled with quality of life after 3 months of ICS/LABA treatment, and in the absence of changes in FEV1.

\section{Further evaluation of airway mechanics}

FOT can be extended to glean further information regarding airway mechanics. By measuring FOT during a maximal inhalation and exhalation, it is possible to evaluate airway distensibility, i.e. the ability of the airways to stretch over a given range of volume [33,34]. Airway distensibility has been found to relate to airway tone [34], as well as asthma control [35]. More recently, the relationship between FOT and lung volume during a slow vital capacity manoeuvre was used to detect the onset of airway closure/lung derecruitment [36]. Furthermore, investigations into deep inspirations have informed the mechanisms behind why asthmatics fail to dilate their airways following airway constriction [37].

\section{Changes during recovery from exacerbation}

FOT may be useful to assess recovery from acute exacerbations of COPD (AECOPD). Out of 29 COPD patients, JETMALANi et al. [38] found that one-third admitted to hospital for an AECOPD exhibited EFL measured by FOT, which was in turn associated with improvement in symptoms and length of stay in hospital. In contrast, STEVENSON et al. [39] detected no change in FOT measurements during recovery from an AECOPD, and instead found increase in inspiratory capacity to be the main determinant and a useful guide of symptom improvement. However, JoHnson et al. [40] showed significant improvement in Xrs 
indices alongside operating lung volume in the 6 weeks following hospital admission and discharge, suggesting Xrs may be a surrogate for the volume changes occurring during recovery from AECOPD.

\section{Home telemonitoring of chronic airway disease}

FOT lends itself particularly well for telemonitoring given its effort-independent nature and ease of performance, and several studies have already demonstrated its feasibility in home telemonitoring of asthma [41] and COPD [42, 43]. In addition to providing a measure of disease status, FOT telemonitoring may further facilitate early, objective detection of exacerbations, which are currently dependent on subjective patient recall and perception of symptoms. Early detection would allow early treatment, which in COPD is known to shorten the duration of AECOPD [44], thus reducing the enormous health and economic burden they impose [2].

A recent large European randomised, interventional trial of FOT home monitoring (CHROMED (Clinical tRials fOr elderly patients with MultiplE Disease)) showed no benefit in time to first hospitalisation or quality of life, though post hoc analyses revealed a significant reduction (-54\%) in repeat hospitalisations in a subset of high-risk patients [43]. This subgroup may represent a particular COPD phenotype that would benefit more from remote monitoring with FOT.

In addition, novel analysis approaches exist [45], which could add value to telemonitoring data by focusing on the day-to-day variability in FOT measurements, known to be increased in asthma and COPD compared with healthy controls [46]. These analyses are based on the idea that biological systems exhibit natural fluctuations which change with disease states and in response to stimuli [32]. They have already shown promise in predicting future deteriorations in lung function in asthma [47] and may help predict risk of future clinically defined exacerbations in both asthma and COPD.

\section{Disease phenotyping and classification}

Asthma and COPD are both highly heterogeneous conditions. In a clinical setting, and in older patients in particular, it can often be challenging to differentiate between these conditions, with prognostic and therapeutic implications [48]. PAREDI et al. [49] applied within-breath FOT analyses to differentiate between asthma and COPD in those with more severe airflow obstruction, demonstrating greater magnitude in $\triangle \mathrm{Xrs}$ in COPD compared to asthma. This finding may reflect the occurrence of dynamic expiratory airway narrowing and closure in COPD, but requires confirmation in larger studies.

The advent of machine learning methods may further help with disease diagnosis and/or phenotyping [50, 51]. AMARAL et al. [52] applied four different machine learning algorithms to FOT measurements from health and COPD, and improved the diagnostic accuracy of using FOT alone in categorising COPD severity. Whether the powerful combination of FOT with such automated, data-driven approaches could aid prediction of disease trajectories and acute exacerbations remains to be seen.

\section{Multiple breath nitrogen washout}

The MBNW test allows measurement of ventilation heterogeneity [53], i.e. unevenness of ventilation distribution within the lungs. It provides information about the efficiency of gas mixing within the lungs, possible mechanisms behind abnormal ventilation distribution and the relative location of underlying pathological processes. The test involves measurement of the concentration of an inert tracer gas (i.e. nitrogen) in the expired breath, which is progressively washed out by inhalation of $100 \%$ oxygen over a series of tidal breaths. If ventilation heterogeneity is increased (i.e. abnormal), there is a delay in clearance of the tracer gas, therefore more breaths will be required to "washout" the nitrogen.

The anatomical and physiological basis of the MBNW test is centred around the principles of gas transport and mixing [54]. Compartmentalisation of ventilation distribution [53, 55-57] allows indices to be derived which reflect ventilation heterogeneity or specific ventilation (ventilation per unit lung volume) within the small airways [58]. Uneven ventilation or differences in specific ventilation in lung units larger than the acini (i.e. the more proximal conducting airways), where gas transport occurs by convection, is termed convection-dependent inhomogeneity (CDI, also known as Scond). In the more distal acinar airways where gas transport occurs by diffusion, it is termed diffusion-convection-dependent inhomogeneity (DCDI, or Sacin) $[57,58]$. Relative contributions to gas mixing from interactions between the convection- and diffusion-dependent airways occur in an intermediate zone and form the "diffusionconvection front" [54]. This quasi-stationary diffusion-convection front determines where CDI and DCDI mechanisms operate and is thought to arise around the acinar entrance in healthy adult lungs [59]. While the terms Scond and Sacin reflect putative locations in the conductive and acinar airways, it is worth noting that these indices are primarily functional rather than anatomical measures.

These indices are calculated from analyses of the MBNW expirogram, i.e. the plot of nitrogen concentration versus expired breath volume for each breath, specifically that portion of the expirogram (also termed 
"phase III") where the expired breath no longer comes from the anatomical dead space and is dominated by alveolar gas alone [53]. A global measure of ventilation heterogeneity, the lung clearance index (LCI), can also be derived from the MBNW test, which describes overall gas mixing efficiency in the lungs.

\section{Relationship with symptoms and disease status}

Ventilation heterogeneity can be abnormal in both asthma [60-62] and COPD [63]. Abnormal Scond and Sacin have been associated with poor asthma control [64]. Both improve with treatment and are also associated with improvement in symptom control [65]. During asthma exacerbations, Scond and Sacin can become more abnormal; however, only $S$ acin is correlated with FEV1 during exacerbations and stable periods [61]. Thus, the acinar airways play a significant role in airflow obstruction in asthma. MBNW indices are also associated with different airway inflammatory profiles in asthma [66]; Scond with airway eosinophilia and Sacin with airway neutrophilia. These associations may have implications on future treatment modalities such as the new biologicals.

Abnormalities measured by MBNW seem to be more pronounced in COPD [67], particularly in Sacin compared to Scond $[62,67]$. In COPD, relationships between Sacin, Scond and different lung function indices have been linked to "acinar lung-zone" and "conductive lung-zone" factors, respectively [63]. Acinar lung-zone factors include lung diffusion capacity; this is consistent with abnormal Sacin and reduced diffusion capacity often seen in COPD, specifically emphysema due to alveolar destruction [68]. A recent modelling study has also demonstrated how depletion of terminal bronchioles leads to an increase in Sacin in COPD, more so when diffusion capacity is low [69]. Conversely, the conductive lung-zone factors include specific airway conductance and forced expiratory flows [63]. This suggests that in COPD, Sacin and Scond reflect alterations of different lung compartments; in contrast to asthma, where an abnormal Sacin may be a sensitive marker of structural alveolar changes, an abnormal Sacin in COPD may involve changes in the proximal acinar airways rather than in the alveoli themselves [62].

Abnormalities in both Sacin and Scond have also been demonstrated in smokers with normal spirometry, with a majority of the abnormalities seen in Sacin [70]. Furthermore, abnormal Sacin, but not Scond, is associated with smoking history and the number of pack-years. These findings point to MBNW as a potential marker of "early" COPD and would support the concept of smoking-related changes beginning in the small airways. This is consistent with studies showing reduced airway count [71] in smokers with normal spirometry, and loss of patent terminal bronchioles occurring even in mild COPD [72], before the onset of emphysematous destruction and beyond with increasing disease severity [73]. Curiously, in smokers who have yet to develop COPD, symptoms of chronic bronchitis were associated with an abnormal Scond but not Sacin [70], suggesting that perhaps structural changes may occur before symptoms manifest.

In smokers with COPD, both Sacin and Scond increase with increasing pack-year smoking history [68]. Following smoking cessation, there is an initial improvement in both Sacin and Scond after 1 week of cessation [74]. However, only Scond shows a persistent improvement after 12 months of smoking cessation with no change in spirometry, diffusion capacity and airway conductance [74]. This suggests early smoking-related lung damage in the acinar airways may be irreversible.

\section{Relationship with airway hyperresponsiveness}

MBNW is also related to other features of airway disease. Airway hyperresponsiveness (AHR) is a hallmark characteristic of asthma, independent of airway inflammation [55]. In healthy subjects, during bronchial provocation testing $S$ acin and Scond worsens [57], i.e. small airways affected by bronchial provocation may be proximal to the acinar airways. However, in those individuals who demonstrated AHR, baseline Sacin was significantly increased compared to those without AHR. Following bronchoprovocation challenge using methacholine, Scond also related to volume of gas trapping [75]. These responses probably reflect overall decrease in airway calibre and heterogeneous narrowing of parallel airways. The relationships between ventilation heterogeneity with AHR and airway closure in asthma have been further corroborated by imaging studies $[76,77]$.

These relationships also appear to be age-dependent, providing insight into the possible interaction between asthma and ageing. In young adults with asthma, Scond is a major determinant of AHR. In contrast, in older people AHR is predicted by Sacin [78], suggesting the acinar rather than conductive airways contribute to ventilation heterogeneity in older people with asthma. It is worth noting that ventilation heterogeneity increases with age in health $[79,80]$, with an accelerated increase seen in Sacin and LCI after the age of 60 years, versus a steady linear increase in Scond [80, 81]. However, Sacin and LCI are both associated with loss of lung elastic recoil in elderly asthma independently of ageing [82]. Alterations to recoil pressure may provide a mechanism for altered distribution of ventilation in these patients, as individual airways are varyingly brought closer to the point of airway closure. 
Evaluation of bronchodilator/treatment response

MBNW has provided insight on whether abnormalities in ventilation heterogeneity are reversible or respond to treatment, and how this varies between different diseases. In asthma, both $S$ cond and $S$ acin improve following administration of SABA [62]. However, abnormal Scond may fail to normalise after a SABA even in mild disease [83].

In contrast, in COPD, despite high levels of abnormality at baseline, Scond and Sacin do not improve following inhalation of a SABA [62]. Similarly, in a study following a 6-week treatment period with tiotropium, ventilation heterogeneity did not improve yet FEV1 and inspiratory capacity did [84]. These COPD subjects also had a low diffusion capacity $[62,84]$, which was consistent with emphysema and may explain the reason for the lack of a significant bronchodilator response. In contrast, in smokers with normal spirometry (i.e. no established COPD), Sacin improved significantly following SABA, and only those with an abnormal baseline Scond demonstrated a significant bronchodilator response [85].

Ventilation heterogeneity is also a known important physiological determinant of response to asthma treatment, with implications for guiding therapy $[65,66,86]$. MBNW indices improve with ICS therapy $[55,65]$ in association with an improvement in symptoms [65]. Ultrafine ICS improves Sacin but not Scond in stable asthma, but only in those whose baseline Sacin was abnormal [86]. Furthermore, worse Scond predicts symptomatic improvement to ICS dose up-titration, which may simply reflect opening up of previously closed conducting airways with ICS. However, worse Sacin predicts worse symptom control during dose down-titration [65]. These findings are useful as they imply that patients who are well-controlled but have abnormal Sacin may not tolerate ICS down-titration.

\section{Current limitations and open questions}

The increasing availability of commercial devices for both FOT and MBNW have supported their ongoing transition from a pure research tool to emerging use in clinical respiratory medicine. Despite the information we have gained about airway disease, made possible by these tests, there still exist gaps in knowledge and limitations that need to be overcome for either test to become commonplace in the clinical evaluation of chronic airways disease.

While international recommendations exist for best practice for both tests [53, 87], issues remain regarding standardisation across centres. There are known differences between equipment, software algorithms and protocols that limit comparability and thus clinical utility, for both FOT [88, 89] and MBNW [90-92]. This lack of comparability has also impacted the availability of reliable and/or widely applicable reference values from a healthy population to derive predicted equations. For FOT, these have either only been collected in specific equipment [93], or there is an inability to pool data across multiple centres [94]. Similarly, reference values exist for MBNW only for specific equipment [60], some of which are custom built by specialised labs and, therefore, are not widely available [79, 80].

In terms of clinical applicability, for both tests there is a need for more rigorously defined cut-off values that define a significant bronchodilator response to aid in diagnosis, as well as identification of minimal clinically important differences to serve as a target for interventions.

Further work into structure-function relationships as higher resolution imaging measures and processing methods become available will add to the insights already gained surrounding the small airways. Most FOT and MBNW studies to date have been limited to smaller mechanistic studies; including those detailed, highly sensitive functional measures in future large cohort studies would enable us to correlate small airway dysfunction with a comprehensive range of biomarkers and clinical outcomes, and add a whole new dimension to clinical phenotyping [95]. Furthermore, coupling these functional measures with inflammatory profiling in interventional studies involving new modalities of treatment (e.g. thermoplasty and the new biologics in asthma) would help elicit a role for small airway dysfunction as a potential treatable trait, in the personalised management of airways disease [96].

\section{Conclusion}

FOT and MBNW are emerging clinical tests that provide novel pulmonary function indices, which have helped us dismantle some of the complex mechanisms underpinning chronic airway diseases. We have demonstrated the knowledge gained and potential clinical utility in the assessment of pulmonary mechanics, response to bronchodilator and other inhaled treatments, the relationship with symptoms and disease status during acute exacerbations and their recovery, and the role in home and clinical monitoring. Overcoming existing limitations, future research and increasing use will further substantiate the role of these tests in clinical evaluation, and their potential in phenotyping and treating chronic airways disease, and personalisation of disease management. 
Author contributions: S.C. Zimmermann, K.O. Tonga and C. Tharmin contributed to the preparation of the manuscript. All authors have read and approved the final manuscript.

Conflict of interest: S.C. Zimmermann has nothing to disclose. K.O. Tonga has nothing to disclose. C. Thamrin has a patent (WO $2006130922 \mathrm{~A} 1$ ) issued and intellectual property arrangements with two FOT device companies, Thorasys Medical Systems and Restech srl relating to scientific collaborations, but does not have any financial relationships with either company.

\section{References}

1 Global Initiative for Asthma (GINA). Global Strategy for Asthma Management and Prevention, 2015. https:// ginasthma.org/wp-content/uploads/2016/01/GINA_Report_2015_Aug11-1.pdf

2 Vogelmeier CF, Criner GJ, Martinez FJ, et al. Global Strategy for the Diagnosis, Management, and Prevention of Chronic Obstructive Lung Disease 2017 Report: GOLD Executive Summary. Eur Respir J 2017; 49: 1700214.

3 van der Wiel E, ten Hacken NHT, Postma DS, et al. Small-airways dysfunction associates with respiratory symptoms and clinical features of asthma: a systematic review. J Allergy Clin Immunol 2013; 131: 646-657.

4 Hogg JC, Paré PD, Hackett T-L. The contribution of small airway obstruction to the pathogenesis of chronic obstructive pulmonary disease. Physiol Rev 2017; 97: 529-552.

5 Usmani OS, Singh D, Spinola M, et al. The prevalence of small airways disease in adult asthma: a systematic literature review. Respir Med 2016; 116: 19-27.

6 O'Donnell DE, Webb KA. The major limitation to exercise performance in COPD is dynamic hyperinflation. J Appl Physiol 2008; 105: 753-755.

7 Hogg JC, Chu F, Utokaparch S, et al. The nature of small-airway obstruction in chronic obstructive pulmonary disease. N Engl J Med 2004; 350: 2645-2653.

8 Sferrazza Papa GF, Pellegrino GM, Pellegrino R. Asthma and respiratory physiology: putting lung function into perspective. Respirology 2014; 19: 960-969.

9 Bates JH, Irvin CG, Farre R, et al. Oscillation mechanics of the respiratory system. Compr Physiol 2011; 1: $1233-1272$.

10 Young HM, Guo F, Eddy RL, et al. Oscillometry and pulmonary MRI measurements of ventilation heterogeneity in obstructive lung disease: relationship to quality of life and disease control. J Appl Physiol 2018; 125: 73-85.

11 Mahadev S, Farah CS, King GG, et al. Obesity, expiratory flow limitation and asthma symptoms. Pulm Pharmacol Ther 2013; 26: 438-443.

12 Eltayara L, Becklake MR, Volta CA, et al. Relationship between chronic dyspnea and expiratory flow limitation in patients with chronic obstructive pulmonary disease. Am J Respir Crit Care Med 1996; 154: 1726-1734.

13 Boni E, Corda L, Franchini D, et al. Volume effect and exertional dyspnoea after bronchodilator in patients with COPD with and without expiratory flow limitation at rest. Thorax 2002; 57: 528-532.

14 Dean J, Kolsum U, Hitchen P, et al. Clinical characteristics of COPD patients with tidal expiratory flow limitation. Int J Chron Obstruct Pulmon Dis 2017; 12: 1503-1506.

15 Dellacà RL, Santus P, Aliverti A, et al. Detection of expiratory flow limitation in COPD using the forced oscillation technique. Eur Respir J 2004; 23: 232-240.

16 Dellacà RL., Rotger M, Aliverti A, et al. Noninvasive detection of expiratory flow limitation in COPD patients during nasal CPAP. Eur Respir J 2006; 27: 983-991.

17 Lorx A, Czövek D, Gingl Z, et al. Airway dynamics in COPD patients by within-breath impedance tracking: effects of continuous positive airway pressure. Eur Respir J 2017; 49: 1601270.

18 Wolkove N, Dajczman E, Colacone A, et al. The relationship between pulmonary function and dyspnea in obstructive lung disease. Chest 1989; 96: 1247-1251.

19 Mahler DA, Faryniarz K, Tomlinson D, et al. Impact of dyspnea and physiologic function on general health status in patients with chronic obstructive pulmonary disease. Chest 1992; 102: 395-401.

20 Wegner RE, Jorres RA, Kirsten DK, et al. Factor analysis of exercise capacity, dyspnoea ratings and lung function in patients with severe COPD. Eur Respir J 1994; 7: 725-729.

21 Frantz S, Nihlen U, Dencker M, et al. Impulse oscillometry may be of value in detecting early manifestations of COPD. Respir Med 2012; 106: 1116-1123.

22 Clement J, Landser FJ, Van de Woestijne KP. Total resistance and reactance in patients with respiratory complaints with and without airways obstruction. Chest 1983; 83: 215-220.

23 Di Mango AM, Lopes AJ, Jansen JM, et al. Changes in respiratory mechanics with increasing degrees of airway obstruction in COPD: detection by forced oscillation technique. Respir Med 2006; 100: 399-410.

24 Yaegashi $M$, Yalamanchili VA, Kaza V, et al. The utility of the forced oscillation technique in assessing bronchodilator responsiveness in patients with asthma. Respir Med 2007; 101: 995-1000.

25 Borrill ZL, Houghton CM, Tal-Singer R, et al. The use of plethysmography and oscillometry to compare long-acting bronchodilators in patients with COPD. Br J Clin Pharmacol 2008; 65: 244-252.

26 Borrill ZL, Houghton CM, Woodcock AA, et al. Measuring bronchodilation in COPD clinical trials. Br J Clin Pharmacol 2005; 59: 379-384.

27 da Costa GM, Faria AC, Di Mango AM, et al. Respiratory impedance and response to salbutamol in healthy individuals and patients with COPD. Respiration 2014; 88: 101-111.

28 Inui N, Matsushima S, Kato S, et al. Effects of indacaterol versus tiotropium on respiratory mechanics assessed by the forced oscillation technique in patients with chronic obstructive pulmonary disease. Int J Chron Obstruct Pulmon Dis 2015; 10: 1139-1146.

29 Diba C, King GG, Berend N, et al. Improved respiratory system conductance following bronchodilator predicts reduced exertional dyspnoea. Respir Med 2011; 105: 1345-1351.

30 Milne S, Hammans C, Watson S, et al. Bronchodilator responses in respiratory impedance, hyperinflation and gas trapping in COPD. COPD 2018; 15: 1-9.

31 Akamatsu T, Shirai T, Shimoda Y, et al. Forced oscillation technique as a predictor of FEV1 improvement in asthma. Respir Physiol Neurobiol 2017; 236: 78-83. 
32 Timmins SC, Diba C, Schoeffel RE, et al. Changes in oscillatory impedance and nitrogen washout with combination fluticasone/salmeterol therapy in COPD. Respir Med 2014; 108: 344-350.

33 Brown NJ, Salome CM, Berend N, et al. Airway distensibility in adults with asthma and healthy adults, measured by forced oscillation technique. Am J Respir Crit Care Med 2007; 176: 129-137.

34 Kelly VJ, Brown NJ, Sands SA, et al. Effect of airway smooth muscle tone on airway distensibility measured by the forced oscillation technique in adults with asthma. J Appl Physiol 2012; 112: 1494-1503.

35 Kelly VJ, Sands SA, Harris RS, et al. Respiratory system reactance is an independent determinant of asthma control. J Appl Physiol 2013; 115: 1360-1369.

36 Nilsen K, Gove K, Thien F, et al. Comparison of two methods of determining lung de-recruitment, using the forced oscillation technique. Eur J Appl Physiol 2018; 118: 2213-2224.

37 Hulme KM, Salome CM, Brown NJ, et al. Deep inspiration volume and the impaired reversal of bronchoconstriction in asthma. Respir Physiol Neurobiol 2013; 189: 506-512.

38 Jetmalani K, Timmins S, Brown NJ, et al. Expiratory flow limitation relates to symptoms during COPD exacerbations requiring hospital admission. Int J Chron Obstruct Pulmon Dis 2015; 10: 939-945.

39 Stevenson NJ, Walker PP, Costello RW, et al. Lung mechanics and dyspnea during exacerbations of chronic obstructive pulmonary disease. Am J Respir Crit Care Med 2005; 172: 1510-1516.

40 Johnson MK, Birch M, Carter R, et al. Measurement of physiological recovery from exacerbation of chronic obstructive pulmonary disease using within-breath forced oscillometry. Thorax 2007; 62: 299-306.

41 Gulotta C, Suki B, Brusasco V, et al. Monitoring the temporal changes of respiratory resistance: a novel test for the management of asthma. Am J Respir Crit Care Med 2012; 185: 1330-1331.

42 Timmins SC, Diba C, Thamrin C, et al. The feasibility of home monitoring of impedance with the forced oscillation technique in chronic obstructive pulmonary disease subjects. Physiol Meas 2013; 34: 67-81.

43 Walker PP, Pompilio PP, Zanaboni P, et al. Telemonitoring in Chronic Obstructive Pulmonary Disease (CHROMED). A randomized clinical trial. Am J Respir Crit Care Med 2018; 198: 620-628.

44 Wilkinson TM, Donaldson GC, Hurst JR, et al. Early therapy improves outcomes of exacerbations of chronic obstructive pulmonary disease. Am J Respir Crit Care Med 2004; 169: 1298-1303.

45 Thamrin C, Zindel J, Nydegger R, et al. Predicting future risk of asthma exacerbations using individua conditional probabilities. J Allergy Clin Immunol 2011; 127: 1494-1502.

46 Timmins SC, Coatsworth N, Palnitkar G, et al. Day-to-day variability of oscillatory impedance and spirometry in asthma and COPD. Respir Physiol Neurobiol 2013; 185: 416-424.

47 Gobbi A, Milesi I, Govoni L, et al. A New Telemedicine System for the Home Monitoring of Lung Function in Patients with Obstructive Respiratory Diseases. 2009 International Conference on eHealth, Telemedicine, and Social Medicine, 2009 1-7 Feb. 2009.

48 Postma DS, Reddel HK, ten Hacken NH, et al. Asthma and chronic obstructive pulmonary disease: similarities and differences. Clin Chest Med 2014; 35: 143-156.

49 Paredi P, Goldman M, Alamen A, et al. Comparison of inspiratory and expiratory resistance and reactance in patients with asthma and chronic obstructive pulmonary disease. Thorax 2010; 65: 263-267.

50 Fernandez-Granero MA, Sanchez-Morillo D, Leon-Jimenez A. An artificial intelligence approach to early predict symptom-based exacerbations of COPD. Biotechnol Biotechnol Equip 2018; 32: 778-784.

51 Pandey G, Pandey OP, Rogers AJ, et al. A nasal brush-based classifier of asthma identified by machine learning analysis of nasal RNA sequence data. Sci Rep 2018; 8: 8826.

52 Amaral JLM, Lopes AJ, Faria ACD, et al. Machine learning algorithms and forced oscillation measurements to categorise the airway obstruction severity in chronic obstructive pulmonary disease. Comput Methods Programs Biomed 2015; 118: 186-197.

53 Robinson PD, Latzin P, Verbanck S, et al. Consensus statement for inert gas washout measurement using multiple-and single-breath tests. Eur Respir J 2013; 41: 507-522.

54 Paiva M. Gas transport in the human lung. J Appl Physiol 1973; 35: 401-410.

55 Downie SR, Salome CM, Verbanck S, et al. Ventilation heterogeneity is a major determinant of airway hyperresponsiveness in asthma, independent of airway inflammation. Thorax 2007; 62: 684-689.

56 Paiva M, Engel LA. Model analysis of gas distribution within human lung acinus. J Appl Physiol Respir Environ Exerc Physiol 1984; 56: 418-425.

57 Verbanck S, Schuermans D, Van Muylem A, et al. Ventilation distribution during histamine provocation. J Appl Physiol 1997; 83: 1907-1916.

58 Crawford AB, Makowska M, Paiva M, et al. Convection- and diffusion-dependent ventilation maldistribution in normal subjects. J Appl Physiol 1985; 59: 838-846.

59 Engel LA. Gas mixing within the acinus of the lung. J Appl Physiol Respir Environ Exerc Physiol 1983; 54: 609-618.

60 Kjellberg S, Houltz BK, Zetterström O, et al. Clinical characteristics of adult asthma associated with small airway dysfunction. Respir Med 2016; 117: 92-102.

61 Thompson BR, Douglass JA, Ellis MJ, et al. Peripheral lung function in patients with stable and unstable asthma. J Allergy Clin Immunol 2013; 131: 1322-1328.

62 Verbanck S, Schuermans D, Noppen M, et al. Evidence of acinar airway involvement in asthma. Am J Respir Crit Care Med 1999; 159: 1545-1550.

63 Verbanck S, Schuermans D, Van Muylem A, et al. Conductive and acinar lung-zone contributions to ventilation inhomogeneity in COPD. Am J Respir Crit Care Med 1998; 157: 5, 1573-1577.

64 Farah CS, King GG, Brown NJ, et al. The role of the small airways in the clinical expression of asthma in adults. J Allergy Clin Immunol 2012; 129: 381-387.

65 Farah CS, King GG, Brown NJ, et al. Ventilation heterogeneity predicts asthma control in adults following inhaled corticosteroid dose titration. J Allergy Clin Immunol 2012; 130: 61-68.

66 Farah CS, Keulers LAB, Hardaker KM, et al. Association between peripheral airway function and neutrophilic inflammation in asthma. Respirology 2015; 20: 975-981.

67 Hardaker KM, Downie SR, Kermode JA, et al. Ventilation heterogeneity is associated with airway responsiveness in asthma but not COPD. Respir Physiol Neurobiol 2013; 189: 106-111.

68 Verbanck S, Schuermans D, Meysman M, et al. Noninvasive assessment of airway alterations in smokers. Am J Respir Crit Care Med 2004; 170: 414-419. 
69 Verbanck S, King GG, Paiva M, et al. The functional correlate of the loss of terminal bronchioles in chronic obstructive pulmonary disease. Am J Respir Crit Care Med 2018; 197: 1633-1635.

70 Jetmalani K, Thamrin C, Farah CS, et al. Peripheral airway dysfunction and relationship with symptoms in smokers with preserved spirometry. Respirology 2018; 23: 512-518.

71 Kirby M, Tanabe N, Tan WC, et al. Total airway count on computed tomography and the risk of chronic obstructive pulmonary disease progression. Findings from a population-based study. Am J Respir Crit Care Med 2018; 197: 56-65.

72 Koo HK, Vasilescu DM, Booth S, et al. Small airways disease in mild and moderate chronic obstructive pulmonary disease: a cross-sectional study. Lancet Respir Med 2018; 6: 591-602.

73 McDonough JE, Yuan R, Suzuki M, et al. Small-airway obstruction and emphysema in chronic obstructive pulmonary disease. N Engl J Med 2011; 365: 1567-1575.

74 Verbanck S, Schuermans D, Paiva M, et al. Small airway function improvement after smoking cessation in smokers without airway obstruction. Am J Respir Crit Care Med 2006; 174: 853-857.

75 King GG, Downie SR, Verbanck S, et al. Effects of methacholine on small airway function measured by forced oscillation technique and multiple breath nitrogen washout in normal subjects. Respir Physiol Neurobiol 2005; 148: 165-177.

76 Farrow CE, Salome CM, Harris BE, et al. Airway closure on imaging relates to airway hyperresponsiveness and peripheral airway disease in asthma. J Appl Physiol 2012; 113: 958-966.

77 Farrow CE, Salome CM, Harris BE, et al. Peripheral ventilation heterogeneity determines the extent of bronchoconstriction in asthma. J Appl Physiol 2017; 123: 1188-1194.

78 Hardaker KM, Downie SR, Kermode JA, et al. Predictors of airway hyperresponsiveness differ between old and young patients with asthma. Chest 2011; 139: 1395-1401.

79 Verbanck S, Thompson BR, Schuermans D, et al. Ventilation heterogeneity in the acinar and conductive zones of the normal ageing lung. Thorax 2012; 67: 789-795.

80 Verbanck S, Van Muylem A, Schuermans D, et al. Transfer factor, lung volumes, resistance and ventilation distribution in healthy adults. Eur Respir J 2016; 47: 166-176.

81 Htun C, Pope A, Lahzami S, et al. The contribution of peripheral airway function to changes in FEVi/FVC and RV/TLC with ageing. J Appl Physiol 2018; 125: 1378-1383.

82 Tonga KO, Berend N, Thamrin C, et al. Lung elastic recoil and ventilation heterogeneity of diffusion-dependent airways in older people with asthma and fixed airflow obstruction. Eur Respir J 2019; 53: 1801028.

83 Verbanck S, Schuermans D, Paiva M, et al. Nonreversible conductive airway ventilation heterogeneity in mild asthma. J Appl Physiol 2003; 94: 1380-1386.

84 Verbanck S, Schuermans D, Vincken W. Small airways ventilation heterogeneity and hyperinflation in COPD: response to tiotropium bromide. Int J Chron Obstruct Pulmon Dis 2007; 2: 625-634.

85 Jetmalani K, Chapman DG, Thamrin C, et al. Bronchodilator responsiveness of peripheral airways in smokers with normal spirometry. Respirology 2016; 21: 1270-1276.

86 Verbanck S, Schuermans D, Paiva M, et al. The functional benefit of anti-inflammatory aerosols in the lung periphery. J Allergy Clin Immunol 2006; 118: 340-346.

87 Oostveen E, MacLeod D, Lorino $\mathrm{H}$, et al. The forced oscillation technique in clinical practice: methodology, recommendations and future developments. Eur Respir J 2003; 22: 1026-1041.

88 Zimmermann SC, Watts JC, Bertolin A, et al. Discrepancy between in vivo and in vitro comparisons of forced oscillation devices. J Clin Monit Comput 2018; 32: 509-512.

89 Watts JC, Farah CS, Seccombe LM, et al. Measurement duration impacts variability but not impedance measured by the forced oscillation technique in healthy, asthma and COPD subjects. ERJ Open Res 2016; 2: 00094-2015.

90 Tonga KO, Robinson PD, Farah CS, et al. In vitro and in vivo functional residual capacity comparisons between multiple- breath nitrogen washout devices. ERJ Open Res 2017; 3: 00011-2017.

91 Yammine S, Singer F, Gustafsson P, et al. Impact of different breathing protocols on multiple-breath washout outcomes in children. J Cyst Fibros 2014; 13, 190-197.

92 Poncin W, Singer F, Aubriot A-S, et al. Agreement between multiple-breath nitrogen washout systems in children and adults. J Cyst Fibros 2017; 16: 258-266.

93 Brown NJ, Xuan W, Salome CM, et al. Reference equations for respiratory system resistance and reactance in adults. Respir Physiol Neurobiol 2010; 172: 162-168.

94 Oostveen E, Boda K, van der Grinten CP, et al. Respiratory impedance in healthy subjects: baseline values and bronchodilator response. Eur Respir J 2013; 42: 1513-1523.

95 Thamrin C, Frey U, Kaminsky DA, et al. Systems biology and clinical practice in respiratory medicine. The twain shall meet. Am J Respir Crit Care Med 2016; 194: 1053-1061.

96 Agusti A, Bel E, Thomas M, et al. Treatable traits: toward precision medicine of chronic airway diseases. Eur Respir J 2016; 47: 359-361. 